\title{
ON THE NUMERICAL RANGE MAP
}

\author{
M. JOSWIG and B. STRAUB
}

(Received 25 November 1997; revised 26 May 1998)

\author{
Communicated by J. A. Hillman
}

\begin{abstract}
Let $A \in \mathscr{L}\left(\mathbb{C}^{n}\right)$ and $A_{1}, A_{2}$ be the unique Hermitian operators such that $A=A_{1}+i A_{2}$. The paper is concerned with the differential structure of the numerical range map $n_{A}: x \longmapsto\left(\left\langle A_{1} x, x\right\rangle,\left\langle A_{2} x, x\right\rangle\right)$ and its connection with certain natural subsets of the numerical range $W(A)$ of $A$. We completely characterize the various sets of critical and regular points of the map $n_{A}$ as well as their respective images within $W(A)$. In particular, we show that the plane algebraic curves introduced by R. Kippenhahn appear naturally in this context. They basically coincide with the image of the critical points of $n_{A}$.
\end{abstract}

1991 Mathematics subject classification (Amer: Math. Soc.): 15A22, 15A60.

\section{Introduction}

Let $\mathbb{C}^{n}$ be the standard $n$-dimensional unitary space equipped with its usual inner product $\langle\cdot, \cdot\rangle$ and norm $\|\cdot\|$. By $\mathscr{L}\left(\mathbb{C}^{n}\right)$ we denote the space of all linear operators of $\mathbb{C}^{n}$ into itself. Associated with each operator $A \in \mathscr{L}\left(\mathbb{C}^{n}\right)$ is the set

$$
W(A)=\left\{\langle A x, x\rangle \mid x \in \mathbb{S}\left(\mathbb{C}^{n}\right)\right\},
$$

where $\mathbb{S}\left(\mathbb{C}^{n}\right)=\left\{x \in \mathbb{C}^{n} \mid\|x\|=1\right\}$, known as the numerical range or the field of values of $A$. This set has been studied extensively (see, for example, $[6,7,8,10,12$, 13, 14]) for it reflects certain important properties of $A$.

One of the main results concerning the geometrical shape of $W(A)$ is due to Kippenhahn [10] (see also [13] and [6]). His investigations in 1951 revealed that

The first and the second author gratefully acknowledge partial and full support, respectively, by the Australian Research Council; this work was carried out at the University of New South Wales.

(C) 1998 Australian Mathematical Society 0263-6115/98 \$A2.00+0.00 
the numerical range of $A$ coincides with the convex hull of a certain plane algebraic curve $C_{A}$ associated with $A$.

On the other hand, the numerical range $W(A)$ is the image of the Rayleigh quotient $R_{A}: x \longmapsto \frac{\langle A x, x\rangle}{\langle x, x\rangle}$, for $x \neq 0$, of $A$. As $W(A)=R_{A}\left(\mathbb{S}\left(\mathbb{C}^{n}\right)\right)$, this yields, for example, that $W(A)$ is always closed and bounded (see, for example, [12, Section 6.6]). However, more attention has been given to the Rayleigh quotient for its differential properties. It is well-known that $R_{A}$ has a stationary value if and only if the operator $A$ and its adjoint $A^{*}$ have an eigenvector in common. In particular, all eigenvectors of $A$ occur in this way if and only if the operator $A$ is normal (see, for example, [14, Section III.18]). In this case, the set of stationary values of $R_{A}$ coincides with the curve $C_{A}$ associated with $A$ as both consist precisely of the $n$ eigenvalues of $A$. This suggests a possible link between the differential structure of $R_{A}$ and the geometrical shape of $W(A)$, especially the curve $C_{A}$.

The aim of this paper is to investigate this connection for an arbitrary (that is, not necessarily normal) operator $A \in \mathscr{L}\left(\mathbb{C}^{n}\right)$. As in [8], we consider the numerical range map $n_{A}: \mathbb{S}\left(\mathbb{R}^{2 n}\right) \longrightarrow \mathbb{R}^{2}$ of $A$ which is given by

$n_{A}(p, q)=\left(\left\langle A_{1}(p+i q), p+i q\right\rangle,\left\langle A_{2}(p+i q), p+i q\right\rangle\right) \quad$ for all $(p, q) \in \mathbb{S}\left(\mathbb{R}^{2 n}\right)$,

Here, $A_{1}$ and $A_{2}$ are the uniquely determined Hermitian operators in $\mathscr{L}\left(\mathbb{C}^{n}\right)$ such that $A=A_{1}+i A_{2}$. By identifying a point $(p, q) \in \mathbb{R}^{n} \times \mathbb{R}^{n} \cong \mathbb{R}^{2 n}$ with the vector $p+i q \in \mathbb{C}^{n}$, the map $n_{A}$ can be viewed as the Rayleigh quotient of $A$ restricted to $\mathbb{S}\left(\mathbb{C}^{n}\right)$.

Following the approach in [8], we split the domain $\$\left(\mathbb{R}^{2 n}\right)$ of $n_{A}$ into the three subsets $\Sigma_{0}(A), \Sigma_{1}(A)$ and $\Sigma_{2}(A)$. Here, $\Sigma_{j}(A)$ is the set of all points $(p, q) \in \mathbb{S}\left(\mathbb{R}^{2 n}\right)$ such that the derivative $n_{A}^{\prime}(p, q)$ of $n_{A}$ at $(\dot{p}, q)$, which is a linear map from the $(2 n-1)$-dimensional tangent space to $\mathbb{S}\left(\mathbb{R}^{2 n}\right)$ at $(p, q)$ into $\mathbb{R}^{2}$, has rank $j$. A new characterization of the sets $\Sigma_{j}(A)$ in terms of properties of the operators $A_{1}$ and $A_{2}$ then allows to extend and refine the results in [8]. For example, it turns out that $n_{A}\left(\Sigma_{2}(A)\right)$, in fact, coincides with the interior of $W(A)$. However, our main result is that the image of the critical points of $n_{A}$, that is, the points where $n_{A}^{\prime}$ does not have the full rank, consists precisely of the curve $C_{A}$ together with all line segments joining points on $C_{A}$ at which $C_{A}$ has the same tangent. This confirms the link between the differential properties of $n_{A}$ and the shape of $W(A)$.

\section{The numerical range map}

Let $A \in \mathscr{L}\left(\mathbb{C}^{n}\right)$. We define Hermitian operators $A_{1}$ and $A_{2}$ in $\mathscr{L}\left(\mathbb{C}^{n}\right)$ as 


$$
A_{1}=\frac{A+A^{*}}{2} \quad \text { and } \quad A_{2}=\frac{A-A^{*}}{2 i}
$$

these are the unique Hermitian operators subject to $A=A_{1}+i A_{2}$.

For each $k=1,2, \ldots$, let $J_{k}: \mathbb{R}^{k} \times \mathbb{R}^{k} \longrightarrow \mathbb{C}^{k}$ be the bijection $J_{k}(p, q)=p+i q$. The Cartesian product $\mathbb{R}^{k} \times \mathbb{R}^{k}$ and the Euclidean space $\mathbb{R}^{2 k}$ are identified in the obvious way.

By $N_{A}$ we denote the map

$$
(p, q) \longmapsto J_{1}^{-1}(\langle A(p+i q), p+i q\rangle), \quad p, q \in \mathbb{R}^{n}
$$

from $\mathbb{R}^{n} \times \mathbb{R}^{n} \cong \mathbb{R}^{2 n}$ into $\mathbb{R}^{2}$. Because $A_{1}$ and $A_{2}$ are Hermitian, both $\left\langle A_{1} x, x\right\rangle$ and $\left\langle A_{2} x, x\right\rangle$ are real numbers for every $x \in \mathbb{C}^{n}$. It follows that

$$
N_{A}(p, q)=\left(\left\langle A_{1}(p+i q), p+i q\right\rangle,\left\langle A_{2}(p+i q), p+i q\right\rangle\right), \quad p, q \in \mathbb{R}^{\prime \prime} .
$$

As the maps $(p, q) \longmapsto\left\langle A_{j}(p+i q), p+i q\right\rangle$ for $j=1,2$ are polynomials in the coordinates of $p$ and $q$ with coefficients in $\mathbb{R}$, it is clear that $N_{A} \in C^{\infty}\left(\mathbb{R}^{2 n}, \mathbb{R}^{2}\right)$. The derivative $N_{A}^{\prime}(p, q)$ of $N_{A}$ at a point $(p, q) \in \mathbb{R}^{2 n}$ is the linear map from $\mathbb{R}^{2 n}$ into $\mathbb{R}^{2}$ specified by the condition

$$
\lim _{(h, k) \rightarrow(0,0)} \frac{N_{A}(p+h, q+k)-N_{A}(p, q)-N_{A}^{\prime}(p, q)(h, k)}{\|(h, k)\|}=0 .
$$

Setting $x=p+i q$, a straightforward calculation shows that $N_{A}^{\prime}(p, q)$ is given by

$$
\begin{aligned}
N_{A}^{\prime}(p, q)(h, k)= & 2\left(\operatorname{Re}\left(\left\langle A_{1} p, h\right\rangle+\left\langle A_{1} q, k\right\rangle\right)+\operatorname{Im}\left(\left\langle A_{1} p, k\right\rangle-\left\langle A_{1} q, h\right\rangle\right),\right. \\
& \left.\quad \operatorname{Re}\left(\left\langle A_{2} p, h\right\rangle+\left\langle A_{2} q, k\right\rangle\right)+\operatorname{Im}\left(\left\langle A_{2} p, k\right\rangle-\left\langle A_{2} q, h\right\rangle\right)\right) \\
= & 2\left(\operatorname{Re}\left\langle A_{1} x, h+i k\right\rangle, \operatorname{Re}\left\langle A_{2} x, h+i k\right\rangle\right)
\end{aligned}
$$

for all $(h, k) \in \mathbb{R}^{2 n}$.

DEFINITION 2.1. The numerical range map $n_{A}$ associated with the operator $A$ in $\mathscr{L}\left(\mathbb{C}^{n}\right)$ is the restriction of $N_{A}$ to the unit sphere $\mathbb{S}\left(\mathbb{R}^{2 n}\right)=\left\{y \in \mathbb{R}^{2 n}:\|y\|=1\right\}$ of $\mathbb{R}^{2 n}$.

It follows that $n_{A}$ is a smooth map from the $(2 n-1)$-dimensional $C^{\infty}$-manifold $\mathbb{S}\left(\mathbb{R}^{2 n}\right)$ into $\mathbb{R}^{2}$. Its derivative $n_{A}^{\prime}(y)$ at a point $y \in \mathbb{S}\left(\mathbb{R}^{2 n}\right)$ is therefore a linear map from the tangent space $T_{y} S\left(\mathbb{R}^{2 n}\right)$ of $\mathbb{S}\left(\mathbb{R}^{2 n}\right)$ at $y$ into $\mathbb{R}^{2}$. Note that $T_{y} \mathbb{S}\left(\mathbb{R}^{2 n}\right)$ is the orthogonal complement $\{y\}^{\perp}$ of the 1 -dimensional subspace $\mathbb{R} y$ in $\mathbb{R}^{2 n}$. More precisely then, the map $n_{A}^{\prime}(y)$ is the restriction of the linear map $N_{A}^{\prime}(y): \mathbb{R}^{2 n} \longrightarrow \mathbb{R}^{2}$ to $\{y\}^{\perp}$. In particular, given $y \in \mathbb{S}\left(\mathbb{R}^{2 n}\right)$, the linear map $n_{A}^{\prime}(y)$ has rank 0 , rank 1 or rank 2 . We split $\mathbb{S}\left(\mathbb{R}^{2 n}\right)$ accordingly and set $\Sigma_{r}(A)=\left\{y \in \mathbb{S}\left(\mathbb{R}^{2 n}\right) \mid \operatorname{rank} n_{A}^{\prime}(y)=r\right\}$ for $r=0,1,2$. 
DEFINITION 2.2. Let $y \in \mathbb{S}\left(\mathbb{R}^{2 n}\right)$. If $y \in \Sigma_{2}(A)$, then we call $y$ a regular point of $n_{A}$. Otherwise $y$ is a critical point of $n_{A}$, and we add rank 0 (rank 1$)$ if we want to specify that $y$ belongs to $\Sigma_{0}(A)\left(\Sigma_{1}(A)\right.$, respectively). A rank 0 critical point is also called a stationary point of $n_{A}$.

REMARK 2.3. An affine transformation of $\mathbb{R}^{2}=\mathbb{C}$ is a map $\tau_{\alpha \beta \gamma}: \mathbb{C} \longrightarrow \mathbb{C}$ with parameters $\alpha, \beta, \gamma \in \mathbb{C}$ such that $\alpha \beta \neq 0$ and $\alpha \beta^{-1} \notin i \mathbb{R}$, which sends $\lambda \in \mathbb{C}$ to $\alpha \operatorname{Re} \lambda+i \beta \operatorname{Im} \lambda+\gamma$. In results about the numerical range of an operator $A$, frequently the corresponding affine transformations $\tau_{\alpha \beta \gamma}(A)=\alpha A_{1}+i \beta A_{2}+\gamma l$ of $A$ are considered (see $[10, \S 2])$ for the numerical range $W\left(\tau_{\alpha \beta \gamma}(A)\right)$ of $\tau_{\alpha \beta \gamma}(A)$ is given by $\tau_{\alpha \beta \gamma}(W(A)),[10, \S 2.4]$. For the derivative of the numerical range map $n_{\tau_{\alpha \beta \gamma}(A)}$ of $\tau_{\alpha \beta \gamma}(A)$ at a point $y \in \mathbb{S}\left(\mathbb{R}^{2 n}\right)$ we obtain

$$
n_{\tau_{\alpha \beta \gamma}(A)}^{\prime}(y)\left(T_{(p, q)} \mathbb{S}\left(\mathbb{R}^{2 n}\right)\right)=J_{1}^{-1}\left(\tau_{\alpha \beta 0}\left(\left(J_{1} \circ n_{A}^{\prime}(y)\right)\left(T_{\langle p . q)} \mathbb{S}\left(\mathbb{R}^{2 n}\right)\right)\right)\right) .
$$

This is an immediate consequence of the representation of $n_{\tau_{\alpha \beta_{Y}}^{\prime}(A)}^{\prime}(y)$. It follows that $\Sigma_{j}\left(\tau_{\alpha \beta \gamma}(A)\right)=\Sigma_{j}(A)$ for $j=0,1,2$.

The proposition below already characterizes the set of all critical points of $n_{A}$.

Proposition 2.4. Let $(c, s) \in \mathbb{R}^{2} \backslash\{(0,0)\}$. Then a point $(p, q) \in \mathbb{S}\left(\mathbb{R}^{2 n}\right)$ is a critical point of $n_{A}$ such that

$$
n_{A}^{\prime}(p, q)\left(T_{(p, q)} \mathbb{S}\left(\mathbb{R}^{2 n}\right)\right) \subseteq \mathbb{R}(c, s)
$$

if and only if the vector $x=p+i q \in \mathbb{S}\left(\mathbb{C}^{n}\right)$ is an eigenvector of the Hermitian operator $s A_{1}-c A_{2}$.

Proof. Assume that $(p, q)$ is a critical point of $n_{A}$ with

$$
n_{A}^{\prime}(p, q)(h, k) \in \mathbb{R}(c, s) \quad \text { for all }(h, k) \in T_{(p, q)} \mathbb{S}\left(\mathbb{R}^{2 n}\right)=\{(p, q)\}^{\perp} .
$$

Since $n_{A}^{\prime}(p, q)(h, k)=2\left(\operatorname{Re}\left\langle A_{1} x, h+i k\right\rangle, \operatorname{Re}\left\langle A_{2} x, h+i k\right\rangle\right)$ and $\mathbb{R}(c, s)=\{(s$, $-c)\}^{\perp}$, this is equivalent to

$$
s \operatorname{Re}\left\langle A_{1} x, h+i k\right\rangle-c \operatorname{Re}\left\langle A_{2} x, h+i k\right\rangle=0 \quad \text { for all }(h, k) \in\{(p, q)\}^{\perp}
$$

which can be written as

$$
\operatorname{Re}\left\langle\left(s A_{1}-c A_{2}\right) x, h+i k\right\rangle=0 \quad \text { for all }(h, k) \in\{(p, q)\}^{\perp}
$$

because $c$ and $s$ are real numbers. However, since

$$
\{(p, q)\}^{\perp}=\left\{(h, k) \in \mathbb{R}^{2 n}: \operatorname{Re}\langle x, h+i k\rangle=0\right\},
$$

it follows that condition (1) holds if and only if $\left(s A_{1}-c A_{2}\right) x \in J_{n}\left(\{(p, q)\}^{\perp \perp}\right)=\mathbb{R} x$, that is, if and only if $x$ is an eigenvector of $s A_{1}-c A_{2}$. 
Proposition 2.4 allows us to distinguish between the two different types of critical points. A point $\lambda=(\beta, \gamma) \in \mathbb{R}^{2}$ is called a joint eigenvalue of a pair $(B, C)$ of operators in $\mathbb{C}^{n}$ if there exists $x \in \mathbb{C}^{\prime \prime} \backslash\{0\}$ such that $B x=\beta x$ and $C x=\gamma x$. In this case, the vector $x$ is called a joint eigenvector of $(B, C)$ corresponding to the joint eigenvalue $\lambda$.

CoRollary 2.5 (see [8, Proposition 1]). The point $(p, q) \in \mathbb{S}\left(\mathbb{R}^{2 n}\right)$ is a stationary point of $n_{A}$ if and only if the vector $x=p+i q$ is a joint eigenvector of some pair $\left(c A_{1}+s A_{2}, d A_{1}+t A_{2}\right)$ with $(c, s),(d, t) \in \mathbb{R}^{2} \backslash\{(0,0)\}$ such that $c t-s d \neq 0$. The corresponding joint eigenvalue is the point $\left(\left\langle\left(c A_{1}+s A_{2}\right) x, x\right\rangle,\left\langle\left(d A_{1}+t A_{2}\right) x, x\right\rangle\right)$.

ProOF. By definition, the point $(p, q)$ is a stationary point if and only if

$$
n_{A}^{\prime}(p, q)\left(T_{(p, q)} \mathbb{S}\left(\mathbb{R}^{2 n}\right)\right)=\{(0,0)\}
$$

Since $\{(0,0)\}=\mathbb{R}(-s, c) \cap \mathbb{R}(-t, d)$, Proposition 2.4 yields that this is equivalent to $x$ being an eigenvector of both operators $c A_{1}+s A_{2}$ and $d A_{1}+t A_{2}$.

We note that if one of the conditions of Corollary 2.5 is satisfied, then the vector $x=p+i q$ is a joint eigenvector of all the pairs $\left(c A_{1}+s A_{2}, d A_{1}+t A_{2}\right)$ with $(c, s),(d, t) \in \mathbb{B}^{2} \backslash\{(0,0)\}$. In particular, then $x$ is a joint eigenvector of the pair $\left(A_{1}, A_{2}\right)$ corresponding to the joint eigenvalue $n_{A}(p, q)$.

Combining Proposition 2.4 and Corollary 2.5 gives the following.

COROLlaRY 2.6. The point $(p, q) \in \mathbb{S}\left(\mathbb{R}^{2 n}\right)$ is a rank 1 critical point of $n_{A}$ if and only if the vector $x=p+i q$ is an eigenvector of $c A_{1}+s A_{2}$ for some point $(c, s) \in \mathbb{R}^{2} \backslash\{(0,0)\}$ which is unique up to multiplication by $\gamma \in \mathbb{R} \backslash\{0\}$.

REMARK 2.7. From its definition it follows that, given $(p, q) \in \mathbb{S}\left(\mathbb{R}^{2 n}\right)$ and $x=$ $p+i q$, the numerical range map $n_{A}$ is constant on the subset $J_{n}^{-1}\left(\mathbb{C} x \cap \mathbb{S}\left(\mathbb{C}^{n}\right)\right)$ of $\mathbb{S}\left(\mathbb{R}^{2 n}\right)$. As a simple consequence of the results above and the fact that multiplication by a complex number $\alpha$ leaves the (complex) eigenspaces of an operator $B \in \mathscr{L}\left(\mathbb{C}^{n}\right)$ invariant, we obtain for the derivative of $n_{A}$ at any of the points $J_{n}^{-1}(\alpha x)$ with $\alpha \in \mathbb{S}(\mathbb{C})$ that

$$
n_{A}^{\prime}\left(J_{n}^{-1}(\alpha x)\right)\left(T_{J_{n}^{-1}(\alpha x)} \mathbb{S}\left(\mathbb{R}^{2 n}\right)\right)=n_{A}^{\prime}(p, q)\left(T_{(p, q)} \mathbb{S}\left(\mathbb{R}^{2 n}\right)\right)
$$

In particular, if $(p, q) \in \mathbb{S}\left(\mathbb{R}^{2 n}\right)$ is a rank 0 (rank 1) critical point of $n_{A}$, then for every $\alpha=\alpha_{1}+i \alpha_{2} \in \mathbb{S}(\mathbb{C})$, the point $J_{n}^{-1}(\alpha(p+i q))=\left(\alpha_{1} p-\alpha_{2} q, \alpha_{1} q+\alpha_{2} p\right)$ is also a rank 0 (rank 1) critical point of $n_{A}$. 
Our main concern in this paper is the connection between certain subsets of the numerical range $W(A)$ of $A$ or, more precisely, of $J_{1}^{-1}(W(A))=n_{A}\left(\mathbb{S}\left(\mathbb{B}^{2 n}\right)\right)$, and the differential structure of the numerical range map $n_{A}$. A first result in this direction was Corollary 2.5. There we have seen that $n_{A}\left(\Sigma_{0}(A)\right)$ is the set of joint eigenvalues of the pair $\left(A_{1}, A_{2}\right)$.

Suppose $(p, q) \in \mathbb{S}\left(\mathbb{R}^{2 n}\right)$ is a point such that $n_{A}(p, q)$ belongs to the boundary $J_{1}^{-1}(\partial W(A))$ of $J_{1}^{-1}(W(A))$. Let $\left\{(a, b) \in \mathbb{R}^{2} \mid c a+s b=\lambda\right\}$ be a supporting line to $J_{1}^{-1}(W(A))$, that is, a line which intersects $J_{1}^{-1}(W(A))$ in a non-empty subset of its boundary, passing through the point $n_{A}(p, q)$. Such a line exists because $W(A)$ is convex. Then $x=p+i q \in \mathbb{S}\left(\mathbb{C}^{n}\right)$ is a vector such that $\left\langle\left(c A_{1}+s A_{2}\right) x, x\right\rangle$ is the maximum or minimum of the set $\left\{\left\langle\left(c A_{1}+s A_{2}\right) z, z\right\rangle \mid z \in \mathbb{S}\left(\mathbb{C}^{\prime \prime}\right)\right\}$. It follows that $x$ is an eigenvector of the operator $c A_{1}+s A_{2}$. Combining this well-known fact (see, for example, [2, Proposition 2.1]) with Proposition 2.4 yields the subsequent result.

\section{LEMMA 2.8. $\quad\left(J_{1} \circ n_{A}\right)^{-1}(\partial W(A)) \subseteq \Sigma_{0}(A) \cup \Sigma_{1}(A)$.}

REMARK 2.9. In the case that $A \in \mathscr{L}\left(\mathbb{C}^{2}\right)$, the inclusion of Lemma 2.8 becomes, in fact, an equality (see also [8, Proposition 4]). For if $(p, q) \in \mathbb{S}\left(\mathbb{R}^{2 n}\right)$ is a critical point of $n_{A}$, then $x=p+i q$ is an eigenvector of some operator $c A_{1}+s A_{2}$ with $(c, s) \in \mathbb{R}^{2} \backslash$ $\{(0,0)\}$ and the corresponding eigenvalue, say $\alpha$, is necessarily the maximal or minimal eigenvalue of $c A_{1}+s A_{2}$. By $[10, \S 3.9-10]$, the line $\left\{(a, b) \in \mathbb{R}^{2} \mid c a+s b=\alpha\right\}$ is a supporting line to $J_{1}^{-1}(W(A))$. Since $n_{A}(p, q)$ is a point on this line, it therefore belongs to $J_{1}^{-1}(\partial W(A))$.

As an immediate consequence of Lemma 2.8 we obtain that $J_{1} \circ n_{A}$ maps all regular points into the interior $W(A)^{\circ}$ of $W(A)$ (see also [8, Proposition 2]). However, as shown below, an even stronger result holds. We make use of the following fact.

REMARK 2.10. Let $X$ be a non-trivial $k$-dimensional subspace of $\mathbb{C}^{n}$ with the induced norm and inner product, and let $P$ be the orthogonal projection of $\mathbb{C}^{n}$ onto $X$. By $\tilde{X}$ we denote the $2 k$-dimensional subspace $J_{n}^{-1}(X)$ of $\mathbb{R}^{2 n}$ corresponding to $X$. Then the operator $\left.P A\right|_{X}$ obtained by restricting the operator $P A$ to $X$, belongs to $\mathscr{L}(X)$, and $\left.P A_{1}\right|_{X}$ and $\left.P A_{2}\right|_{X}$ are the unique Hermitian operators in $\mathscr{L}(X)$ such that $\left.P A\right|_{X}=\left.P A_{1}\right|_{X}+\left.i P A_{2}\right|_{X}$. The numerical range map $n_{P A_{X X}}$ of $\left.P A\right|_{X}$ is a smooth map from the unit sphere $S(\tilde{X})$ of $\tilde{X}$ into $\mathbb{R}^{2}$. In fact, $n_{P A \mid X}$ is the restriction of $n_{A}$ to the $(2 k-1)$-dimensional $C^{\infty}$-submanifold $\mathbb{S}(\tilde{X})$ of $\mathbb{S}\left(\mathbb{R}^{2 n}\right)$. It follows that the derivative $n_{P A \mid X}^{\prime}(y)$ of $n_{\left.P A\right|_{X}}$ at a point $y \in \mathbb{S}(\tilde{X})$ is the restriction of $n_{A}^{\prime}(y)$ to the tangent space $T_{y} \mathbb{S}(\tilde{X})$ of $\mathbb{S}(\tilde{X})$ at $y$. That is, the linear map $n_{P A \mid x}^{\prime}(y)$ is the restriction of $N_{A}^{\prime}(y)$ to the orthogonal complement $\{y\}^{\perp} \cap \tilde{X}$ of $\mathbb{R} y$ in $\tilde{X}$. In particular, $\operatorname{rank} n_{P A \mid x}^{\prime}(y) \leq \operatorname{rank} n_{A}^{\prime}(y)$ for all $y \in \mathbb{S}(\tilde{X})$. 
We write $l_{c, s, \lambda}$ for the line $\left\{(a, b) \in \mathbb{R}^{2} \mid c a+s b=\lambda\right\}$ in $\mathbb{R}^{2}$ and, as this requires implicitly that $(c, s) \neq(0,0)$, we adopt as a convention that the coordinates $(c, s, \lambda)$ satisfy $c=\cos \varphi, s=\sin \varphi$ for some angle $\varphi \in[0, \pi)$.

PROPOSITION 2.11. $\quad n_{A}\left(\Sigma_{2}(A)\right)=J_{1}^{-1}\left(W(A)^{\circ}\right)$

PROOF. It remains to show that every point in $J_{1}^{-1}\left(W(A)^{\circ}\right)$ which is the image of a critical point is also the image of a regular point of $n_{A}$. Note that such a point can not exist if $\mathbb{C}^{n}$ has dimension $n \leq 2$ (see Remark 2.9).

Let $\mu_{0} \in J_{1}^{-1}\left(W(A)^{\circ}\right) \cap n_{A}\left(\Sigma_{0}(A) \cup \Sigma_{1}(A)\right)$ and let $L$ be the set of lines $l_{c . s . \lambda}$ through the point $\mu_{0}$ with coordinates $(c, s, \lambda)$ such that $\lambda$ is an eigenvalue of the operator $c A_{1}+s A_{2}$. We pick a line $l_{c_{0}, s_{0}, \lambda_{0}}$ through the point $\mu_{0}$ such that $l_{c_{0}, s_{0}, \lambda_{0}} \notin L$, if such a line exists. Otherwise we take a line $l_{c_{0}, s_{0}, \lambda_{0}} \in L$ satisfying $l_{c_{0}, s_{0}, \lambda_{0}} \cap n_{A}\left(\Sigma_{0}(A)\right) \subseteq$ $\left\{\mu_{0}\right\}$.

Without loss of generality we can assume that $c_{0}=1$ and $s_{0}=0$. If not, consider the operator $B=e^{-i \varphi_{0}} A$, where $\varphi_{0} \in[0, \pi)$ is the angle such that $\cos \varphi_{0}=c_{0}$ and $\sin \varphi_{0}=s_{0}$. By Remark 2.3, we have $\Sigma_{j}(B)=\Sigma_{j}(A)$ for $j=0,1,2$.

The line $l_{1,0 . \lambda_{0}}$ intersects the boundary of $J_{1}^{-1}(W(A))$ in two points $\mu_{1}$ and $\mu_{2}$. For $j=1,2$, take $\left(p_{j}, q_{j}\right) \in n_{A}^{-1}\left(\left\{\mu_{j}\right\}\right)$ and put $x_{j}=p_{j}+i q_{j}$. Note that, by Lemma 2.8 and the choice of $l_{1.0 . \lambda_{0}}$, the two points $\left(p_{1}, q_{1}\right)$ and $\left(p_{2}, q_{2}\right)$ belong to $\Sigma_{1}(A)$. Let $X$ be the 2-dimensional subspace of $\mathbb{C}^{n}$ spanned by the vectors $x_{1}$ and $x_{2}$. Then, using the notation of Remark 2.10, the numerical range $J_{1}^{-1}\left(W\left(\left.P A\right|_{X}\right)\right)$ of $\left.P A\right|_{X}$ is either an ellipse or a line segment.

If $J_{1}^{-1}\left(W\left(\left.P A\right|_{X}\right)\right)=n_{\left.P A\right|_{X}}(S(\tilde{X}))$ is an ellipse, then, by Remark 2.9 , its interior is the image of the set of regular points of $n_{\left.P A\right|_{X}}$. In particular, there exists a regular point $\left(p_{0}, q_{0}\right)$ of $n_{P A \mid X}$ in $\mathbb{S}(\tilde{X}) \subseteq \mathbb{S}\left(\mathbb{R}^{2 n}\right)$ such that $n_{\left.P A\right|_{X}}\left(p_{0}, q_{0}\right)=\mu_{0}$. By Remark 2.10, $\left(p_{0}, q_{0}\right)$ is also a regular point of $n_{A}$ and $n_{A}\left(p_{0}, q_{0}\right)=\mu_{0}$.

Suppose $J_{1}^{-1}\left(W\left(\left.P A\right|_{X}\right)\right)$ is a line segment. More precisely then, it is the line segment joining the points $\mu_{1}$ and $\mu_{2}$ and contained in the line $l_{1.0 . \lambda_{0}}$. This implies $\left.P A_{1}\right|_{X}=\lambda_{0} I_{X}$ because $\left\langle\left(\left.P A_{1}\right|_{X}-\lambda_{0} I_{X}\right) x, x\right\rangle=0$ for all $x \in X$. It follows further that every point $\left(p_{0}, q_{0}\right) \in n_{P A \mid x}^{-1}\left(\left\{\mu_{0}\right\}\right)$ is a rank 1 critical point of $n_{\left.P A\right|_{X}}$ and, by Proposition 2.4, that $n_{P A \mid X}^{\prime}\left(p_{0}, q_{0}\right)\left(T_{\left(p_{0} . q_{0}\right)} \$(\tilde{X})\right)=\mathbb{R}(0,1)=l_{1,0.0}$. Remark 2.10 yields that $n_{A}^{\prime}\left(p_{0}, q_{0}\right)\left(T_{\left(p_{0}, q_{0}\right)} \mathbb{S}\left(\mathbb{R}^{2 n}\right)\right)$ contains at least the line $l_{1,0.0}$.

If $l_{1.0 . \lambda_{0}} \notin L$, then we can deduce from Proposition 2.4 that every point $\left(p_{0}, q_{0}\right) \in$ $n_{P A \mid X}^{-1}\left(\left\{\mu_{0}\right\}\right)$ is a regular point of $n_{A}$. Hence, assume that $l_{1,0, \lambda_{0}} \in L$. Since $\left.P A_{2}\right|_{X}$ is Hermitian and $\mu_{1} \neq \mu_{2}$, the vectors $x_{1}$ and $x_{2}$ are eigenvectors of $P A_{2} \mid x$ and form an orthonormal basis of $X$. Thus, a point $(p, q) \in n_{p_{A \mid x}}^{-1}\left(\left\{\mu_{0}\right\}\right)$ is of the form $J_{n}^{-1}\left(\alpha x_{1}+\beta x_{2}\right)$ for constants $\alpha, \beta \in \mathbb{C}$ satisfying $|\alpha|^{2}+|\beta|^{2}=1$ and $n_{P_{A \mid X}}(p, q)=$ $\left(\lambda_{0},|\alpha|^{2}\left\langle A_{2} x_{1}, x_{1}\right\rangle+|\beta|^{2}\left\langle A_{2} x_{2}, x_{2}\right\rangle\right)=\mu_{0}$. It suffices to show that there exists $\left(h_{0}, k_{0}\right) \in \tilde{X}^{\perp}$ such that $\operatorname{Re}\left\langle A_{1} x_{1}, h_{0}+i k_{0}\right\rangle \neq 0$. Then we can find $\alpha_{0}, \beta_{0} \in \mathbb{C}$ 
satisfying $\left(p_{0}, q_{0}\right)=J_{n}^{-1}\left(\alpha_{0} x_{1}+\beta_{0} x_{2}\right) \in n_{P A \mid x}^{-1}\left(\left\{\mu_{0}\right\}\right)$ and $\operatorname{Re}\left\langle A_{1}\left(\alpha_{0} x_{1}+\beta_{0} x_{2}\right), h_{0}+\right.$ $\left.i k_{0}\right\rangle \neq 0$. As $\left(h_{0}, k_{0}\right) \in\left\{\left(p_{0}, q_{0}\right)\right\}^{\perp}$ and $n_{A}^{\prime}\left(p_{0}, q_{0}\right)$ is given by $n_{A}^{\prime}\left(p_{0}, q_{0}\right)(h, k)=$ $2\left(\operatorname{Re}\left\langle A_{1}\left(\alpha_{0} x_{1}+\beta_{0} x_{2}\right), h+i k\right\rangle, \operatorname{Re}\left\langle A_{2}\left(\alpha_{0} x_{1}+\beta_{0} x_{2}\right), h+i k\right\rangle\right)$ for all $(h, k) \in$ $T_{\left(p_{1:}, q_{0}\right)} \lessgtr\left(\mathbb{R}^{2 n}\right)=\left\{\left(p_{0}, q_{0}\right)\right\}^{\perp}$, this implies that $n_{A}^{\prime}\left(p_{0}, q_{0}\right)\left(T_{\left(p_{0}, q_{0}\right)} \lessgtr\left(\mathbb{R}^{2 n}\right)\right)$ contains a point $\left(r_{1}, r_{2}\right)$ with $r_{1} \neq 0$ in addition to the line $l_{1,0.0}$, and therefore the whole plane. In other words, $\left(p_{0}, q_{0}\right)$ is a regular point of $n_{A}$ such that $n_{A}\left(p_{0}, q_{0}\right)=\mu_{0}$.

Now, since $\left(p_{1}, q_{1}\right)$ is a rank 1 critical point of $n_{A}$ and $l_{1.0 . \lambda_{0}}$ is not the supporting line to $J_{1}^{-1}(W(A))$ at $\mu_{1}$, there exists a pair $(c, s) \in \mathbb{S}\left(\mathbb{R}^{2}\right)$ with $s>0$ such that $n_{A}^{\prime}\left(p_{1}, q_{1}\right)\left(T_{\left(p_{1}, q_{1}\right)} \mathbb{S}\left(\mathbb{R}^{2 n}\right)\right)=l_{c, s, 0}$. On the other hand, $\left(p_{1}, q_{1}\right) \in \Sigma_{0}\left(\left.P A\right|_{X}\right)$ which gives $n_{A}^{\prime}\left(p_{1}, q_{1}\right)\left(T_{\left(p_{1}, q_{1}\right)} \mathbb{S}(\tilde{X})\right)=\{(0,0)\}$. As $T_{\left(p_{1}, q_{1}\right)} \mathbb{S}(\tilde{X})=\left\{\left(p_{1}, q_{1}\right)\right\}^{\perp} \cap \tilde{X}$, there exists a point $\left(h_{0}, k_{0}\right) \in \tilde{X}^{\lrcorner}$and a real number $\gamma \neq 0$ such that $n_{A}^{\prime}\left(p_{1}, q_{1}\right)\left(h_{0}, k_{0}\right)=$ $\gamma(-s, c)$. In particular, $\operatorname{Re}\left\langle A_{1} x_{1}, h_{0}+i k_{0}\right\rangle=-\gamma s \neq 0$. This completes the proof.

The worst case in the proof of Proposition 2.11 is indeed possible, that is, it can happen that every line through an interior point which is the image of a critical point, belongs to $L$ and that the corresponding operator $\left.P A\right|_{X}$ has a segment of the line for its numerical range. We illustrate this with an example.

EXAMPLE 2.12. Consider the operator $A=A_{1}+i A_{2} \in \mathscr{L}\left(\mathbb{C}^{3}\right)$, where $A_{1}$ and $A_{2}$ are the Hermitian operators

$$
A_{1}=\left(\begin{array}{ccc}
0 & \frac{1}{2} & 0 \\
\frac{1}{2} & 0 & \frac{1}{2} \\
0 & \frac{1}{2} & 0
\end{array}\right) \quad \text { and } \quad A_{2}=\left(\begin{array}{ccc}
1 & 0 & 0 \\
0 & 0 & 0 \\
0 & 0 & -1
\end{array}\right) \text {. }
$$

The numerical range $W(A)$ of $A$ is the ellipse $\left\{\lambda \in \mathbb{C}: 2(\operatorname{Re} \lambda)^{2}+(\operatorname{Im} \lambda)^{2} \leq 1\right\}$, and the numerical range map $n_{A}$ is given by

$$
n_{A}(p, q)=\left(p_{2}\left(p_{1}+p_{3}\right)+q_{2}\left(q_{1}+q_{3}\right), p_{1}^{2}-p_{3}^{2}+q_{1}^{2}-q_{3}^{2}\right) \quad \text { for }(p, q) \in \mathbb{S}\left(\mathbb{R}^{6}\right)
$$

A direct calculation shows that $\Sigma_{1}(A)$ consists of the two sets $S_{1}=\{(a v, b v) \mid v \in$ $\left.\mathbb{S}\left(\mathbb{R}^{3}\right), v_{2}^{2}=2 v_{1} v_{3}, \quad(a, b) \in \mathbb{S}\left(\mathbb{R}^{2}\right)\right\}$ and $S_{2}=\left\{(a v, b v) \mid v \in \mathbb{S}\left(\mathbb{R}^{3}\right), v_{1}=-v_{3}\right.$, $\left.(a, b) \in \mathbb{S}\left(\mathbb{R}^{2}\right)\right\}$. All other points $(p, q) \in \mathbb{S}\left(\mathbb{R}^{6}\right)$ belong to $\Sigma_{2}(A)$. In particular, $\Sigma_{0}(A)=\emptyset$. The image of $S_{1}$ under $n_{A}$ is precisely the boundary of $J_{1}^{-1}(W(A))$, whereas $n_{A}\left(S_{2}\right)=\{(0,0)\}$. We note that 0 is an eigenvalue of every operator $c A_{1}+s A_{2}$ with $(c, s) \in \mathbb{R}^{2} \backslash\{(0,0)\}$, that is, all lines through the point $(0,0)$ belong to the set $L$ of Proposition 2.11 .

Any line $l$ through the point $(0,0) \in \mathbb{R}^{2}$ has a representation $\left\{(a, b) \in \mathbb{R}^{2} \mid(2 t-1) a\right.$ $-\sqrt{2 t(1-t)} b=0\}$ for some $0<t \leq 1$. It intersects $J_{1}^{-1}(\partial W(A))$ in the two points $\mu_{1}(t)=(\sqrt{2 t(1-t)}, 2 t-1)$ and $\mu_{2}(t)=-\mu_{1}(t)$. For $j=1,2$, we have 
$n_{A}^{-1}\left(\left\{\mu_{j}\right\}\right)=J_{3}^{-1}\left(\mathbb{C} x_{j}(t) \cap \mathbb{S}\left(\mathbb{C}^{3}\right)\right)$, where the vectors $x_{1}(t), x_{2}(t) \in \mathbb{C}^{3}$ are given by

$$
x_{1}(t)=\left(\begin{array}{c}
t \\
\sqrt{2 t(1-t)} \\
1-t
\end{array}\right) \quad \text { and } \quad x_{2}(t)=\left(\begin{array}{c}
1-t \\
-\sqrt{2 t(1-t)} \\
t
\end{array}\right) .
$$

Let $X(t)=\mathbb{C} x_{1}(t)+\mathbb{C} x_{2}(t), \tilde{X}(t)=J_{3}^{-1}(X(t))$ and let $P(t)$ be the orthogonal projection of $\mathbb{C}^{3}$ onto $X(t)$. For $j, k=1,2$, the tangent space $T_{J_{3}^{-1}\left(x_{j}(t)\right)} \$(\tilde{X}(t))=$ $\left\{J_{3}^{-1}\left(x_{j}(t)\right)\right\}^{\perp} \cap \tilde{X}(t)$ of $\mathbb{S}(\tilde{X}(t))$ at $J_{3}^{-1}\left(x_{j}(t)\right)$ is spanned by the three points in $J_{3}^{-1}\left(\left\{i x_{j}(t), x_{3-j}(t), i x_{3-j}(t)\right\}\right)$, and $\operatorname{Re}\left\langle A_{k} x_{j}(t), x\right\rangle=0$ for every vector $x \in\left\{i x_{j}(t)\right.$, $\left.x_{3-j}(t), i x_{3-j}(t)\right\}$. It follows that the points $J_{3}^{-1}\left(x_{1}(t)\right)$ and $J_{3}^{-1}\left(x_{2}(t)\right)$ in $\$(\tilde{X}(t))$ are stationary points of the numerical range map $n_{\left.P(t) A\right|_{(t)}}$ of $\left.P(t) A\right|_{X(t)}$, and, by Remark 2.7 , that $n_{A}^{-1}\left(\left\{\mu_{1}(t)\right\}\right) \cup n_{A}^{-1}\left(\left\{\mu_{2}(t)\right\}\right)=\Sigma_{0}\left(\left.P(t) A\right|_{X(t)}\right)$. Hence, $J_{1}^{-1}\left(W\left(\left.P(t) A\right|_{X(t)}\right)\right)$ is the line segment joining the points $\mu_{1}(t)$ and $\mu_{2}(t)$.

We note that the point $(0,0) \in \mathbb{R}^{2}$ is, for example, the image under $n_{A}$ of the regular point $\left(\left(\begin{array}{c}\frac{\sqrt{2}}{2} \\ 0 \\ 0\end{array}\right),\left(\begin{array}{c}0 \\ 0 \\ \frac{\sqrt{2}}{2}\end{array}\right)\right)$. This point was obtained by taking $t=1$ in the above and proceeding then as in the proof of Proposition 2.11.

In the remaining part of the paper, we will have a closer look at the image of the rank 1 critical points of $n_{A}$. Motivated by the observations of Remark 2.7, we take a geometric approach.

\section{Geometric properties}

Let $\mathbb{K} \in\{\mathbb{R}, \mathbb{C}\}$ and $m \in \mathbb{N}$. The Grassmannian $G_{m . k} \mathbb{K}$ is the set of all $k$ dimensional $\mathbb{K}$-subspaces of the vector space $\mathbb{K}^{m}$. It is a compact analytic $\mathbb{K}$-manifold of dimension $(m-k) k$. The space $G_{m, k} \mathbb{K}$ has a natural topology which is induced by the differential structure of the manifold. This topology is, for example, determined by the metric $h$ on $G_{m, k} \mathbb{K}$ with

$$
h(U, V)=\sup _{v \in V \cap S\left(\mathbb{K}^{m}\right)} \inf _{u \in U \cap S\left(\mathbb{K}^{m}\right)}\|u-v\| \quad \text { for all } U, V \in G_{m, k} \mathbb{K} .
$$

The metric $h$ corresponds to the Hausdorff metric (see [5, 4.5.23]) on the set $B_{m . k}=$ $\left\{U \cap \mathbb{S}\left(\mathbb{K}^{m}\right) \mid U \in G_{m, k} \mathbb{K}\right\}$. Other ways of describing the topology on $\mathrm{G}_{m, k} \mathbb{K}$ can be found, for example, in [17, Chapter 13] or [4, VII.8.2].

The $(m-1)$-dimensional projective space $\mathrm{PG}\left(\mathbb{K}^{m}\right)$ over $\mathbb{K}$ is defined as

$$
\operatorname{PG}\left(\mathbb{K}^{m}\right)=\bigcup_{0 \leq k \leq m} G_{m \cdot k} \mathbb{K} .
$$


There are geometric operations $\vee$ and $\wedge$ defined on $\operatorname{PG}\left(\mathbb{K}^{m}\right)$, where $U \vee V$ and $U \wedge V$ denote the span and the intersection of the subspaces $U, V \leq \mathbb{K}^{m}$, respectively.

Usually, the 1-, 2-, $(m-1)$-dimensional subspaces of $\mathbb{K}^{m}$ are called the points, lines, and hyperplanes in $\mathrm{PG}\left(\mathbb{K}^{m}\right)$, respectively. The span of two points is a line and the intersection of two hyperplanes is an element of $G_{m, m-2} \mathbb{K}$.

By common abuse of notation we introduce homogeneous coordinates for the points in $\operatorname{PG}\left(\mathbb{K}^{m}\right)$ as $\left(u_{1}: \ldots: u_{m}\right)=\mathbb{K}\left(u_{1}, \ldots, u_{m}\right)$ whenever it is clear which field $\mathbb{K}$ is involved. The coordinates of a vector in $\mathbb{K}^{m}$ are always expressed with respect to a fixed (standard) basis of $\mathbb{K}^{m}$.

A polarity $\pi$ of $\mathrm{PG}\left(\mathbb{K}^{m}\right)$ is a bijection on $\mathrm{PG}\left(\mathbb{K}^{m}\right)$ which reverses the inclusion of subspaces and satisfies $\pi^{2}=$ id. Throughout the paper, $\pi$ denotes the standard polarity of $\mathrm{PG}\left(\mathbb{K}^{m}\right)$ which is characterized by

$$
u^{\pi}=\left\{v \in \mathbb{K}^{m} \mid \sum_{j=1}^{m} u_{j} v_{j}=0\right\} \quad \text { for all } u \in G_{m, 1} \mathbb{K} .
$$

It follows that $u^{\pi} \in G_{m, m-1} \mathbb{K}$. Using the polarity $\pi$ we can also introduce homogeneous coordinates on $G_{m, m-1} \mathbb{K}$ by setting $\left[v_{1}: \ldots: v_{m}\right]=\left(v_{1}: \ldots: v_{m}\right)^{\pi}$.

In the special case where $m=3$, the projective space $\operatorname{PG}\left(\mathbb{K}^{3}\right)$ is called the projective plane over $\mathbb{K}$. Here lines are the same as hyperplanes.

From Remark 2.7 it is clear that the numerical range map induces a mapping which is defined on a complex projective space of the proper dimension.

DEFINITION 3.1. For $A \in \mathscr{L}\left(\mathbb{C}^{n}\right)$, we define the map $v_{A}$ from $G_{n, 1} \mathbb{C}$ into $G_{3,1} \mathbb{R}$ by

$$
\nu_{A}(\mathbb{C} x)=\mathbb{R}\left(\left\langle A_{1} x, x\right\rangle,\left\langle A_{2} x, x\right\rangle,\|x\|^{2}\right) .
$$

As $\|x\| \neq 0$ for $x \neq 0$, we infer that the image of $v_{A}$ is contained in an affine subplane $F$ of $\mathrm{PG}\left(\mathbb{R}^{3}\right)$ with the point set $\left\{\left(\alpha_{1}: \alpha_{2}: 1\right) \mid\left(\alpha_{1}, \alpha_{2}\right) \in \mathbb{R}^{2}\right\}$. Let $\tau: F \longrightarrow \mathbb{R}^{2}$ be the bijection $\tau\left(\alpha_{1}: \alpha_{2}: 1\right)=\left(\alpha_{1}, \alpha_{2}\right)$. By Remark 2.7 , the numerical range map $n_{A}$ from $\mathbb{S}\left(\mathbb{R}^{2 n}\right) \cong \mathbb{S}\left(\mathbb{C}^{n}\right)$ to $\mathbb{R}^{2}$ factors through the projective space $\operatorname{PG}\left(\mathbb{C}^{n}\right)$. The factoring map is given by $v_{A}$. More precisely, we have

$$
n_{A}=\tau \circ v_{A} \circ \sigma,
$$

where $\sigma: \mathbb{S}\left(\mathbb{R}^{2 n}\right) \longrightarrow G_{n, 1} \mathbb{C}$ is the map $\sigma(p, q)=\mathbb{C}(p+i q)$ for all $(p, q) \in \mathbb{S}\left(\mathbb{R}^{2 n}\right)$.

A non-empty subset $C$ of $G_{3,1} \mathbb{K}$ is called a plane $\mathbb{K}$-algebraic curve if it is the zero locus of a homogeneous 3-variate polynomial over $\mathbb{K}$. We note that the defining polynomial of $C$ is not uniquely determined; for example, if $f$ defines the curve, then so does $f^{k}$ for any $k \geq 1$. A curve is said to be irreducible if it has an irreducible defining polynomial. Since a polynomial ring over a field is a unique factorization 
domain, each algebraic curve $C$ is the union of finitely many irreducible curves. If $C_{1}, \ldots, C_{k}$ are the irreducible components of $C$ with irreducible defining polynomials $f_{1}, \ldots, f_{k}$, then the polynomial $f=f_{1} \cdots f_{k}$ is a defining polynomial of $C$ of minimal degree. We call $f$ a minimal polynomial of $C$. It is unique up to a constant factor. Note that an irreducible real algebraic curve is not necessarily connected.

Let $C=\left\{u \in G_{3,1} \mathbb{K} \mid f(u)=0\right\}$ be an algebraic curve and let $f$ be a minimal polynomial of $C$. A point $u \in C$ is called singular or a singularity of $C$ if $\left(\partial f / \partial u_{j}\right)(u)=0$ for $j=1,2,3$. Observe that $C$ has only finitely many singular points. These are the singular points of the irreducible components of $C$ together with the points of intersection of any two of these components. A non-singular point $u \in C$ is called a simple point of $C$. The curve $C$ is the topological closure of its simple points. Also, if $u \in C$ is simple, then there exists a neighborhood of $u$ in which $C$ admits a smooth parameterization.

Let $C$ be an irreducible plane algebraic curve and $f$ be its minimal polynomial. At each simple point $u \in C$, we have a unique tangent line to $C$ which is given by

$$
\mathscr{T}_{u} C=\left[\frac{\partial f}{\partial u_{1}}(u): \frac{\partial f}{\partial u_{2}}(u): \frac{\partial f}{\partial u_{3}}(u)\right] .
$$

If $C$ is not a projective line or a point, then it is well known that the set $\left\{\left(\mathscr{T}_{u} C\right)^{\pi}\right\}$ $u \in C$ simple\} is contained in a unique irreducible algebraic curve $C^{*}$, the so-called dual curve of $C$. As an algebraic curve has at most finitely many singularities, the dual curve is the topological closure of the set $\left\{\left(\mathscr{T}_{u} C\right)^{\pi} \mid u \in C\right.$ simple $\}$. We have $C^{* *}=C$. If $C$ is a projective line, then $\left\{\left(\mathscr{T}_{u} C\right)^{\pi} \mid u \in C\right\}$ consists of a single point $u$ in PG $\left(\mathbb{K}^{3}\right)$. In this case, we set $C^{*}=\{u\}$ and define $C^{* *}$ to be the image under $\pi$ of the set of all lines in $\mathrm{PG}\left(\mathbb{K}^{3}\right)$ which pass through $u$. Then again we have $C^{* *}=C$. The dual curve of a general plane algebraic curve $C$ is the union of the dual curves of its irreducible components. In particular, $C$ and $C^{*}$ have the same number of irreducible components.

Naturally, the general theory of real algebraic curves differs quite a bit from the complex theory. However, the above statements on the duality of real algebraic curves immediately follow from the corresponding statements in the complex case.

The details and further information on complex algebraic curves can be found, for example, in [16]. The literature for the real case is somewhat less easy to access. As a general reference to the theory of real algebraic geometry, see [3].

Let $A \in \mathscr{L}\left(\mathbb{C}^{n}\right)$. We define an algebraic curve $C_{A}$ in the real projective plane by setting its dual curve to be

$$
D_{A}=\left\{(c: s: \lambda) \in G_{3,1} \mathbb{R} \mid \operatorname{det}\left(c A_{1}+s A_{2}+\lambda I\right)=0\right\} .
$$

We write $f_{A}$ for the defining polynomial of $D_{A}$, that is, $f_{A}(c, s, \lambda)=\operatorname{det}\left(c A_{1}+s A_{2}+\right.$ $\lambda I)$ for $(c: s: \lambda) \in G_{3,1} \mathbb{R}$, and denote the minimal polynomial of $D_{A}$ by $m_{A}$. 
Kippenhahn [10] showed that the curve $C_{A}=D_{A}^{*}$ is contained in the affine subplane $F=\left\{\left(\alpha_{1}: \alpha_{2}: 1\right) \mid\left(\alpha_{1}, \alpha_{2}\right) \in \mathbb{R}^{2}\right\}$ and that the convex hull $\operatorname{co}\left(C_{A}\right)$ of $C_{A}$ is exactly the image of $\nu_{A}$, that is, $\tau\left(\operatorname{co}\left(C_{A}\right)\right)$ is the numerical range of $A$. Note that $C_{A}$ and $D_{A}$ are the real parts of the curves in $\operatorname{PG}\left(\mathbb{C}^{3}\right)$ considered in [10]. Our aim is to show that the curve $C_{A}$ itself is contained in the image under $\tau^{-1} \circ n_{A}=\nu_{A} \circ \sigma$ of the set $\Sigma_{0}(A) \cup \Sigma_{1}(A)$ of critical points of $n_{A}$.

Every point $u \in D_{A}$ has a representation $(\cos \varphi: \sin \varphi: \lambda)$ for some $\varphi \in[0, \pi)$ and $\lambda \in \mathbb{R}$. As $u$ is a zero of $f_{A}$, it follows that $-\lambda$ is an eigenvalue of the operator $\mathscr{A}(\varphi)=\cos \varphi A_{1}+\sin \varphi A_{2}$.

LEMMA 3.2. Let the map $\mathscr{A}: \mathbb{R} \longrightarrow \mathscr{L}\left(\mathbb{C}^{n}\right)$ be given by $\mathscr{A}(\varphi)=\cos \varphi A_{1}+$ $\sin \varphi A_{2}$ for $\varphi \in \mathbb{R}$. Let $\varphi_{0} \in \mathbb{R}$ and $\lambda_{0}$ be an eigenvalue with multiplicity $r$ of the operator $\mathscr{A}\left(\varphi_{0}\right)$. Then there exists a neighborhood $T$ of $\varphi_{0}$ and regular analytic functions $\lambda_{j}: T \longrightarrow \mathbb{R}$ and $x_{j}: T \longrightarrow \mathbb{S}\left(\mathbb{C}^{n}\right)$ with $1 \leq j \leq r$, such that $\lambda_{j}\left(\varphi_{0}\right)=\lambda_{0}$, $\mathscr{A}(\varphi) x_{j}(\varphi)=\lambda_{j}(\varphi) x_{j}(\varphi)$ and $\left\langle x_{j}(\varphi), x_{k}(\varphi)\right\rangle=\delta_{j k}$ for every $\varphi \in T$ and $1 \leq j, k \leq r$. Moreover, for every $1 \leq j \leq r$, the derivative of $\lambda_{j}(\cdot)$ at $\varphi_{0}$ is given by $\lambda_{j}^{\prime}\left(\varphi_{0}\right)=$ $\left\langle\mathscr{A}^{\prime}\left(\varphi_{0}\right) x_{j}\left(\varphi_{0}\right), x_{j}\left(\varphi_{0}\right)\right\rangle$.

PROOF. The map $\mathscr{A}$ is a regular analytic function from $\mathbb{R}$ into the Hermitian operators on $\mathbb{C}^{n}$. We can thus apply $[15$, Satz 1$]$ and obtain the functions $\lambda_{j}(\cdot)$ and $x_{j}(\cdot)$ with the desired properties.

For $\varphi \in T$ we can express $\lambda_{j}(\varphi)$ as $\left\langle\mathscr{A}(\varphi) x_{j}(\varphi), x_{j}(\varphi)\right\rangle$. Then

$$
\begin{aligned}
\lambda_{j}^{\prime}\left(\varphi_{0}\right)= & \lim _{h \rightarrow 0} \frac{\left\langle\mathscr{A}\left(\varphi_{0}+h\right) x_{j}\left(\varphi_{0}+h\right), x_{j}\left(\varphi_{0}+h\right)\right\rangle-\left\langle\mathscr{A}\left(\varphi_{0}\right) x_{j}\left(\varphi_{0}\right), x_{j}\left(\varphi_{0}\right)\right\rangle}{h} \\
= & \lim _{h \rightarrow 0} \frac{\sin h}{h}\left\langle\left(-\sin \varphi_{0} A_{1}+\cos \varphi_{0} A_{2}\right) x_{j}\left(\varphi_{0}+h\right), x_{j}\left(\varphi_{0}+h\right)\right\rangle \\
& +\lim _{h \rightarrow 0} \frac{\cos h-1}{h}\left\langle\mathscr{A}\left(\varphi_{0}\right) x_{j}\left(\varphi_{0}+h\right), x_{j}\left(\varphi_{0}+h\right)\right\rangle \\
& +\lim _{h \rightarrow 0} \frac{\left\langle\mathscr{A}\left(\varphi_{0}\right) x_{j}\left(\varphi_{0}+h\right), x_{j}\left(\varphi_{0}+h\right)\right\rangle-\left\langle\mathscr{A}\left(\varphi_{0}\right) x_{j}\left(\varphi_{0}\right), x_{j}\left(\varphi_{0}\right)\right\rangle}{h} \\
= & L_{1}+L_{2}+L_{3} .
\end{aligned}
$$

The continuity of $x_{j}(\cdot)$ in $\varphi_{0}$ gives $L_{1}=\left\langle\mathscr{A}^{\prime}\left(\varphi_{0}\right) x_{j}\left(\varphi_{0}\right), x_{j}\left(\varphi_{0}\right)\right\rangle$ and $L_{2}=0$.

As $\mathscr{A}\left(\varphi_{0}\right)=\mathscr{A}\left(\varphi_{0}\right)+i 0$, we can apply the results of Section 2 to the third limit. Let $P_{1}: \mathbb{R}^{2} \longrightarrow \mathbb{R}$ be the projection onto the first coordinate and let $p(\varphi)=$ $J_{n}^{-1}\left(x_{j}(\varphi)\right) \in \mathbb{R}^{2 n}$ for $\varphi \in T$. Then

$$
\begin{gathered}
\frac{\left\langle\mathscr{A}\left(\varphi_{0}\right) x_{j}\left(\varphi_{0}+h\right), x_{j}\left(\varphi_{0}+h\right)\right\rangle-\left\langle\mathscr{A}\left(\varphi_{0}\right) x_{j}\left(\varphi_{0}\right), x_{j}\left(\varphi_{0}\right)\right\rangle}{h} \\
=P_{1}\left(\frac{n_{\mathscr{A}\left(\varphi_{0}\right)}\left(p\left(\varphi_{0}+h\right)\right)-n_{\mathscr{A}\left(\varphi_{0}\right)}\left(p\left(\varphi_{0}\right)\right)}{h}\right)
\end{gathered}
$$




$$
=P_{1}\left(\frac{n_{\mathscr{A}\left(\varphi_{0}\right)}^{\prime}\left(p\left(\varphi_{0}\right)\right)\left(p\left(\varphi_{0}+h\right)-p\left(\varphi_{0}\right)\right)+o\left(p\left(\varphi_{0}+h\right)-p\left(\varphi_{0}\right)\right)}{h}\right),
$$

where $o$ is the Landau symbol. Since $x_{j}\left(\varphi_{0}\right)$ is a joint eigenvector of the pair $\left(\mathscr{A}\left(\varphi_{0}\right), 0\right)$, it follows by Corollary 2.5 that $n_{\mathscr{A}\left(\varphi_{0}\right)}^{\prime}\left(p\left(\varphi_{0}\right)\right)=0 \in \mathscr{L}\left(\mathbb{R}^{2 n}, \mathbb{R}^{2}\right)$. Hence

$$
L_{3}=P_{1}\left(\lim _{h \rightarrow 0} \frac{o\left(p\left(\varphi_{0}+h\right)-p\left(\varphi_{0}\right)\right)}{\left\|p\left(\varphi_{0}+h\right)-p\left(\varphi_{0}\right)\right\|} \frac{\left\|p\left(\varphi_{0}+h\right)-p\left(\varphi_{0}\right)\right\|}{h}\right)=0
$$

and

$$
\lambda_{j}^{\prime}\left(\varphi_{0}\right)=\left\langle\mathscr{A}^{\prime}\left(\varphi_{0}\right) x_{j}\left(\varphi_{0}\right), x_{j}\left(\varphi_{0}\right)\right\rangle
$$

We can now prove the first main result in this section.

PROPOSITION 3.3. Let $A \in \mathscr{L}\left(\mathbb{C}^{n}\right)$. Then the curve $C_{A}$ is contained in the image under $v_{A} \circ \sigma$ of the critical points of $n_{A}$.

PRoOF. Let $M$ be the (finite) set of singularities of $D_{A}$, and $u_{0}=\left(\cos \varphi_{0}: \sin \varphi_{0}\right.$ : $\lambda_{0}$ ) be a point in $D_{A} \backslash M$. Then $u_{0}$ belongs to a uniquely determined irreducible component $D$ of $D_{A}$, and there exists a neighborhood $N$ of $u_{0}$ such that every point $u \in D_{A} \cap N$ is contained in $D \backslash M$. As $u_{0}$ is a zero of $f_{A},-\lambda_{0}$ is an eigenvalue of $\mathscr{A}\left(\varphi_{0}\right)$, say with multiplicity $r$. By Lemma 3.2, there exist $r$ functions $\varphi \longmapsto \lambda_{j}(\varphi)$ defined and regular analytic in a neighborhood $T$ of $\varphi_{0}$, such that $\lambda_{j}\left(\varphi_{0}\right)=\lambda_{0}$ and $-\lambda_{j}(\varphi)$ is an eigenvalue of the operator $\mathscr{A}(\varphi)$ for every $\varphi \in T$ and $1 \leq j \leq r$. It follows that in a neighborhood $N_{0} \subseteq N$ of $u_{0}$, the curve $D$ has the $r$ parameterizations $\varphi \mapsto\left(\cos \varphi: \sin \varphi: \lambda_{j}(\varphi)\right)$ for $\varphi \in T_{0} \subseteq T$. As none of the points in $D \cap N_{0}$ belongs to $M$, this gives $\lambda_{j}(\varphi)=\lambda_{1}(\varphi)$ for all $\varphi \in T_{0}$ and $1 \leq j \leq r$. In particular, for every $\varphi \in T_{0}$ the eigenspace of $\mathscr{A}(\varphi)$ corresponding to $-\lambda_{1}(\varphi)$ has dimension $r$. Then the tangent to $D_{A}$ at the point $\left(\cos \varphi_{0}: \sin \varphi_{0}: \lambda_{1}\left(\varphi_{0}\right)\right)$ is given by the formula

$$
\begin{aligned}
& \left(\cos \varphi_{0}: \sin \varphi_{0}: \lambda_{1}\left(\varphi_{0}\right)\right) \vee\left(-\sin \varphi_{0}: \cos \varphi_{0}: \lambda_{1}^{\prime}\left(\varphi_{0}\right)\right) \\
& \quad=\left[-\cos \varphi_{0} \lambda_{1}\left(\varphi_{0}\right)+\sin \varphi_{0} \lambda_{1}^{\prime}\left(\varphi_{0}\right):-\sin \varphi_{0} \lambda_{1}\left(\varphi_{0}\right)-\cos \varphi_{0} \lambda_{1}^{\prime}\left(\varphi_{0}\right): 1\right] .
\end{aligned}
$$

By Lemma 3.2, there exists a regular analytic function $x: T_{0} \longrightarrow \mathbb{S}\left(\mathbb{C}^{n}\right)$ such that $\mathscr{A}(\varphi) x(\varphi)=-\lambda_{1}(\varphi) x(\varphi)$ for all $\varphi \in T_{0}$. Moreover, we have $\lambda_{1}\left(\varphi_{0}\right)=$ $-\left\langle\mathscr{A}\left(\varphi_{0}\right) x\left(\varphi_{0}\right), x\left(\varphi_{0}\right)\right\rangle$ and $\lambda_{1}^{\prime}\left(\varphi_{0}\right)=-\left\langle\mathscr{A}^{\prime}\left(\varphi_{0}\right) x\left(\varphi_{0}\right), x\left(\varphi_{0}\right)\right\rangle$.

It follows that the tangent to $D_{A}$ at the point $\left(\cos \varphi_{0}: \sin \varphi_{0}: \lambda_{0}\right)$ is given by

$$
\begin{aligned}
& {\left[\cos \varphi_{0}\left\langle\mathscr{A}\left(\varphi_{0}\right) x\left(\varphi_{0}\right), x\left(\varphi_{0}\right)\right\rangle-\sin \varphi_{0}\left\langle\mathscr{A}^{\prime}\left(\varphi_{0}\right) x\left(\varphi_{0}\right), x\left(\varphi_{0}\right)\right\rangle:\right.} \\
& \left.\sin \varphi_{0}\left\langle\mathscr{A}\left(\varphi_{0}\right) x\left(\varphi_{0}\right), x\left(\varphi_{0}\right)\right\rangle+\cos \varphi_{0}\left\langle\mathscr{A}^{\prime}\left(\varphi_{0}\right) x\left(\varphi_{0}\right), x\left(\varphi_{0}\right)\right\rangle: 1\right] \\
& =\left[\left\langle A_{1} x\left(\varphi_{0}\right), x\left(\varphi_{0}\right)\right\rangle:\left\langle A_{2} x\left(\varphi_{0}\right), x\left(\varphi_{0}\right)\right\rangle: 1\right]
\end{aligned}
$$


Its image under $\pi$, that is, the point $\left(\left\langle A_{1} x\left(\varphi_{0}\right), x\left(\varphi_{0}\right)\right\rangle:\left\langle A_{2} x\left(\varphi_{0}\right), x\left(\varphi_{0}\right)\right\rangle: 1\right) \in C_{A}$, is the image under $\nu_{A} \circ \sigma=\tau^{-1} \circ n_{A}$ of the critical point $p\left(\varphi_{0}\right)=J_{n}^{-1}\left(x\left(\varphi_{0}\right)\right)$.

Let $S$ be the set of points of $C_{A}$ which correspond to the tangents to $D_{A}$ at simple points. Note that $S$ contains all components of $C_{A}$ which consist of single points. We have shown above that all points $u \in S$ belong to $\left(v_{A} \circ \sigma\right)\left(\Sigma_{0}(A) \cup \Sigma_{1}(A)\right)$. However, as $D_{A}$ has only finitely many singular points and none of these is a component of $D_{A}$, the set $C_{A} \backslash S$ is at most finite. It follows that $\tau\left(C_{A}\right)$ is the closure of $\tau(S)$ in $\mathbb{R}^{2}$. Since $n_{A}\left(\Sigma_{0}(A) \cup \Sigma_{1}(A)\right)$ is a closed set in $\mathbb{R}^{2}$ which contains $\tau(S), \tau\left(C_{A}\right)$ must be a subset of $n_{A}\left(\Sigma_{0}(A) \cup \Sigma_{1}(A)\right)$ as well. Hence $C_{A} \subseteq\left(\nu_{A} \circ \sigma\right)\left(\Sigma_{0}(A) \cup \Sigma_{1}(A)\right)$.

From the proof of Proposition 3.3 it is clear that the curve $C_{A}$ coincides with the image of the critical points of $n_{A}$ if $f_{A}=m_{A}$ and $D_{A}$ is a curve without singularities. In order to clarify the general situation we need the following lemma.

LEMMA 3.4. Suppose $\left(\cos \varphi_{0}: \sin \varphi_{0}: \lambda_{0}\right)$ is a point of $D_{A}$. Let $E$ be the eigenspace of the operator $\mathscr{A}\left(\varphi_{0}\right)$ corresponding to its eigenvalue $-\lambda_{0}$ and $P$ be the orthogonal projection of $\mathbb{C}^{n}$ onto $E$. Let $K$ be the set of points of $C_{A}$ at which the line $\left[\cos \varphi_{0}\right.$ : $\left.\sin \varphi_{0}: \lambda_{0}\right]$ is tangent to $C_{A}$. Then $\left(J_{1} \circ \tau\right)(K)$ is the set of eigenvalues of the operator $\left.P A\right|_{E}$, and $\left(n_{A} \circ J_{n}^{-1}\right)(E)$ is the convex hull of $\tau(K)$.

ProOF. Let $r$ be the dimension of $E$, and let $\lambda_{j}(\cdot)$ and $x_{j}(\cdot)$ with $1 \leq j \leq r$ be the regular analytic eigenvalue and eigenvector functions as given by Lemma 3.2. Then in a neighborhood of $\left(\cos \varphi_{0}: \sin \varphi_{0}: \lambda_{0}\right)$, the curve $D_{A}$ has the $r$ (not necessarily distinct) parameterizations $\Phi_{j}: \varphi \longmapsto\left(\cos \varphi: \sin \varphi:-\lambda_{j}(\varphi)\right)$. Following the argument in the proof of Proposition 3.3, we infer that for $1 \leq j \leq r$, the projective line $\left[\left\langle A_{1} x_{j}\left(\varphi_{0}\right), x_{j}\left(\varphi_{0}\right)\right\rangle:\left\langle A_{2} x_{j}\left(\varphi_{0}\right), x_{j}\left(\varphi_{0}\right)\right\rangle: 1\right]$ is the tangent to $D_{A}$ at the point $\left(\cos \varphi_{0}: \sin \varphi_{0}: \lambda_{0}\right)$ along the parameterization $\Phi_{j}$. The duality of the algebraic curves $C_{A}$ and $D_{A}$ yields that the line $\left[\cos \varphi_{0}: \sin \varphi_{0}: \lambda_{0}\right]$ is a tangent to the curve $C_{A}$ at the point $\left(\left\langle A_{1} x_{j}\left(\varphi_{0}\right), x_{j}\left(\varphi_{0}\right)\right\rangle:\left\langle A_{2} x_{j}\left(\varphi_{0}\right), x_{j}\left(\varphi_{0}\right)\right\rangle: 1\right)$. This can be verified by computing the tangent directly using that, in a neighborhood of the point $\left(\left\langle A_{1} x_{j}\left(\varphi_{0}\right), x_{j}\left(\varphi_{0}\right)\right\rangle\right.$ : $\left.\left\langle A_{2} x_{j}\left(\varphi_{0}\right), x_{j}\left(\varphi_{0}\right)\right\rangle: 1\right)$, the curve $C_{A}$ admits the parameterization

$$
\varphi \longmapsto\left(\cos \varphi \lambda_{j}(\varphi)-\sin \varphi \lambda_{j}^{\prime}(\varphi): \sin \varphi \lambda_{j}(\varphi)+\cos \varphi \lambda_{j}^{\prime}(\varphi): 1\right)
$$

(see equation (2)). In particular, the duality of $C_{A}$ and $D_{A}$ yields that $K$ is the set $\left\{\left(\left\langle A_{1} x_{j}\left(\varphi_{0}\right), x_{j}\left(\varphi_{0}\right)\right\rangle:\left\langle A_{2} x_{j}\left(\varphi_{0}\right), x_{j}\left(\varphi_{0}\right)\right\rangle: 1\right) \mid 1 \leq j \leq r\right\}$.

It follows that the $r$ vectors $x_{j}\left(\varphi_{0}\right)$, which form an orthonormal basis of $E$, belong to $\left(J_{n} \circ n_{A}^{-1} \circ \tau\right)(K)$. They are eigenvectors of the operator $\left.P \mathscr{A}^{\prime}\left(\varphi_{0}\right)\right|_{E}$. In fact, by Lemma 3.2, we have $\lambda_{j}^{\prime}\left(\varphi_{0}\right)=\left\langle\mathscr{A}^{\prime}\left(\varphi_{0}\right) x_{j}\left(\varphi_{0}\right), x_{j}\left(\varphi_{0}\right)\right\rangle$ for every $1 \leq j \leq r$. By [11, Theorem 7], the derivatives $\lambda_{j}^{\prime}\left(\varphi_{0}\right)$ are the eigenvalues of the $(r \times r)$-matrix $\left(\left\langle\mathscr{A}^{\prime}\left(\varphi_{0}\right) x_{j}\left(\varphi_{0}\right), x_{k}\left(\varphi_{0}\right)\right\rangle\right)_{1 \leq j, k \leq r}$. Hence $\left\langle\mathscr{A}^{\prime}\left(\varphi_{0}\right) x_{j}\left(\varphi_{0}\right), x_{k}\left(\varphi_{0}\right)\right\rangle=0$ for all $j \neq k$. 
This implies $n_{B}^{\prime}\left(J_{n}^{-1}\left(x_{j}\left(\varphi_{0}\right)\right)\right)=0$ for all $1 \leq j \leq r$, where $B$ denotes the operator $\left.e^{-i \varphi_{0}} P A\right|_{E}=\left.P \mathscr{A}\left(\varphi_{0}\right)\right|_{E}+\left.i P \mathscr{A}^{\prime}\left(\varphi_{0}\right)\right|_{E}$. By Corollary 2.5 , the $r$ vectors $x_{j}\left(\varphi_{0}\right)$ are joint eigenvectors of the pairs $\left(\left.P \mathscr{A}\left(\varphi_{0}\right)\right|_{E},\left.P \mathscr{A}^{\prime}\left(\varphi_{0}\right)\right|_{E}\right)$ and $\left(\left.P A_{1}\right|_{E},\left.P A_{2}\right|_{E}\right)$. Since $\left(n_{A} \circ J_{n}^{-1}\right)(E)=J_{1}^{-1}\left(W\left(\left.P A\right|_{E}\right)\right)$, this completes the proof.

From Lemma 3.4 we can deduce that if the curve $D_{A}$ has a unique tangent at the point $\left(\cos \varphi_{0}: \sin \varphi_{0}: \lambda_{0}\right)$, then $n_{A} \circ J_{n}^{-1}$ maps the eigenspace $E$ of the operator $\mathscr{A}\left(\varphi_{0}\right)$ corresponding to its eigenvalue $-\lambda_{0}$ to a single point in $\mathbb{R}^{2}$ which belongs to $\tau\left(C_{A}\right)$. This follows from the fact that then $K$ consists of a single point; the condition holds, in particular, for all simple points of $D_{A}$. If there exists more than one line which is tangent to $D_{A}$ at $\left(\cos \varphi_{0}: \sin \varphi_{0}: \lambda_{0}\right)$ along some local parameterization of $D_{A}$, then the set $K$ contains more than one point. In this case, $\left(n_{A} \circ J_{n}^{-1}\right)(E)$ is the segment of the line $l_{\cos \varphi_{0}, \sin \varphi_{0},-\lambda_{0}}$ which joins all points in $\tau(K)$. We have thus proved the following theorem.

THEOREM 3.5. Let $A \in \mathscr{L}\left(\mathbb{C}^{n}\right)$. The image under $v_{A} \circ \sigma$ of the critical points of $n_{A}$ consists of the curve $C_{A}$ together with all line segments joining pairs of points of $C_{A}$ at which $C_{A}$ has the same tangent line.

If $\left(\lambda_{1}, \lambda_{2}\right)$ belongs to $n_{A}\left(\Sigma_{0}(A)\right)$, then $\left(\lambda_{1}, \lambda_{2}\right)$ is a joint eigenvalue of the pair $\left(A_{1}, A_{2}\right)$. It follows that $\left(u \lambda_{1}+v \lambda_{2}+w\right)$ is a factor of $f_{A}$ and so $\left\{\left(\lambda_{1}: \lambda_{2}: 1\right)\right\}$ is a component of $C_{A}$. From Theorem 3.5 one therefore obtains the image of the rank 1 critical points of $n_{A}$ by subtracting all such components of $C_{A}$. However, as is well known, a component of $C_{A}$ which consists only of a single point does not necessarily correspond to a joint eigenvalue of the pair $\left(A_{1}, A_{2}\right)$. In Example 2.12, for instance, $\{(0: 0: 1)\}$ is a component of $C_{A}$, yet the set of stationary points is empty.

From the local representation of $C_{A}$ given above, one can derive formulas for the curvature of $\tau\left(C_{A}\right)$ at all but a finite number of points.

PROPOSITION 3.6. Let $\left(u_{0}, v_{0}, 1\right) \in C_{A}$, and $\left[\cos \varphi_{0}, \sin \varphi_{0}, \lambda_{0}\right]$ be a tangent to $C_{A}$ at $\left(u_{0}, v_{0}, 1\right)$. If $\left(\cos \varphi_{0}, \sin \varphi_{0}, \lambda_{0}\right)$ is a simple point of $D_{A}$, then the signed curvature $k_{0}$ of $\tau\left(C_{A}\right)$ at the point $\left(u_{0}, v_{0}\right)$ is given by

$$
k_{0}=\left|\frac{2}{r} \operatorname{trace}\left(\mathscr{A}^{\prime}\left(\varphi_{0}\right) S \mathscr{A}^{\prime}\left(\varphi_{0}\right) P\right)\right|^{-1},
$$

where $r$ is the multiplicity of the eigenvalue $-\lambda_{0}$ of $\mathscr{A}\left(\varphi_{0}\right), P$ is the orthogonal projection onto the eigenspace of $\mathscr{A}\left(\varphi_{0}\right)$ corresponding to $-\lambda_{0}$ and $S$ denotes the reduced resolvent (or Moore-Penrose inverse) of $\mathscr{A}\left(\varphi_{0}\right)+\lambda_{0}$.

Proof. Suppose $\left(\cos \varphi_{0}, \sin \varphi_{0}, \lambda_{0}\right)$ is a simple point of $D_{A}$. Then by Lemma 3.2 there exists a unique local eigenvalue function $\lambda(\cdot)$ such that $\lambda\left(\varphi_{0}\right)=-\lambda_{0}$. From 
equation (2) it follows that in a neighborhood of $\left(u_{0}, v_{0}\right)$ the curve $\tau\left(C_{A}\right)$ admits the parameterization $\varphi \longmapsto(u(\varphi), v(\varphi))$, say for $\varphi \in T_{0}$, where $u(\varphi)=\cos \varphi \lambda(\varphi)-$ $\sin \varphi \lambda^{\prime}(\varphi)$ and $v(\varphi)=\sin \varphi \lambda(\varphi)+\cos \varphi \lambda^{\prime}(\varphi)$.

The signed curvature $k(\varphi)$ at any point $(u(\varphi), v(\varphi))$ with $\varphi \in T_{0}$ can then be computed by the formula

$$
k(\varphi)=\frac{u^{\prime}(\varphi) v^{\prime \prime}(\varphi)-u^{\prime \prime}(\varphi) v^{\prime}(\varphi)}{\left(u^{\prime}(\varphi)^{2}+v^{\prime}(\varphi)^{2}\right)^{3 / 2}}
$$

This gives $k_{0}=\left|\lambda\left(\varphi_{0}\right)+\lambda^{\prime \prime}\left(\varphi_{0}\right)\right|^{-1}$ (see [1, p. 295]).

From the perturbation results of [9, Section II.2], applied to the map $\psi \longmapsto \mathscr{A}(\psi+$ $\left.\varphi_{0}\right)=\cos (\psi) \mathscr{A}\left(\varphi_{0}\right)+\sin (\psi) \mathscr{A}^{\prime}\left(\varphi_{0}\right)$, we obtain a power series expansion of the eigenvalue function $\psi \longmapsto \tilde{\lambda}(\psi)=\lambda\left(\psi+\varphi_{0}\right)$ at $\psi=0$, that is, $\tilde{\lambda}(\psi)=\sum_{k=0}^{\infty} \Lambda_{k} \psi^{k}$. For the details on the coefficients $\Lambda_{k}$, we refer to [9, Section II.2]. It follows that $\lambda^{\prime \prime}\left(\varphi_{0}\right)=\tilde{\lambda}^{\prime \prime}(0)=2 \Lambda_{2}=\frac{2}{r} \operatorname{trace}\left(-\frac{1}{2} \mathscr{A}\left(\varphi_{0}\right) P-\mathscr{A}^{\prime}\left(\varphi_{0}\right) S \mathscr{A}^{\prime}\left(\varphi_{0}\right) P\right)$ with $P$ and $S$ as above. This completes the proof.

Note that $k_{0}$ is allowed to be infinity; this situation will occur, for example, if $\left\{\left(u_{0}, v_{0}, 1\right)\right\}$ is a component of $C_{A}$.

REMARK 3.7. Let $x(\cdot)$ be any of the $r$ (local) eigenvector functions $x_{j}(\cdot)$ obtained in Lemma 3.2. Then we have $\lambda(\varphi)=\langle\mathscr{A}(\varphi) x(\varphi), x(\varphi)\rangle$ and $\lambda^{\prime}(\varphi)=$ $\left\langle\mathscr{A}^{\prime}(\varphi) x(\varphi), x(\varphi)\right\rangle$ for all $\varphi$ in the domain of $x(\cdot)$. Using this representation of $\lambda^{\prime}(\varphi)$, an argument similar to the one applied in the proof of Lemma 3.2 yields that $\lambda^{\prime \prime}(\varphi)=-\lambda(\varphi)+2 \operatorname{Re}\left\langle\mathscr{A}^{\prime}(\varphi) x(\varphi), x^{\prime}(\varphi)\right\rangle$. Hence, the curvature of $\tau\left(C_{A}\right)$ at $\left(x_{0}, y_{0}\right)$ is also given by

$$
k_{0}=\left|2 \operatorname{Re}\left\langle\mathscr{A}^{\prime}\left(\varphi_{0}\right) x\left(\varphi_{0}\right), x^{\prime}\left(\varphi_{0}\right)\right\rangle\right|^{-1}
$$

REMARK 3.8. Proposition 3.6 yields in particular the curvature of the numerical range $W(A)$ at certain boundary points. Let $\left(u_{0}, v_{0}\right) \in \partial W(A)$. If there exists a supporting line $l_{c, s, \lambda}$ to $\partial W(A)$ at $\left(u_{0}, v_{0}\right)$ such that $(c, s,-\lambda)$ is a simple point of $D_{A}$, then the curvature of $\partial W(A)$ at $\left(u_{0}, v_{0}\right)$ is given by the formulas above. A sufficient but not necessary condition for $(c, s,-\lambda)$ to be a simple point of $D_{A}$ is that $\lambda$ is a simple eigenvalue of the operator $c A_{1}+s A_{2}$. In this case, our formula for $k_{0}$ is the reciprocal of the corresponding formula for the radius of curvature derived from $[6$, Theorem 3.3]. Thus, Proposition 3.6 is the natural generalization of [6, Theorem 3.3]. 


\section{References}

[1] J. Bazer and D. H. Y. Yen, 'Lacunas of the Riemann matrix of symmetric-hyperbolic systems in two space variables', Comm. Pure Appl. Math. 22 (1969), 279-333.

[2] P. Binding and C-K. Li, 'Joint ranges of Hermitian matrices and simultaneous diagonalization', Linear Algebra Appl. 151 (1991), 157-167.

[3] J. Bochnak, M. Coste and M.-F. Roy, Géométrie Algébrique Réelle (Springer, New York, 1987).

[4] G. E. Bredon, Topology and Geometry, Graduate Texts in Mathematics 139 (Springer, New York, 1993).

[5] R. Engelking, General Topology, 2nd edition (Heldermann, Berlin, 1992).

[6] M. Fiedler, 'Geometry of the numerical range of matrices', Linear Algebra Appl. 37 (1981), 81-96.

[7] _ - 'Numerical range of matrices and Levinger's theorem', Linear Algebra Appl. 220 (1995), $171-180$.

[8] J. A. Hillman, B. R. F. Jefferies, W. J. Ricker and B. Straub, 'Differential properties of the numerical range map of pairs of matrices', Linear Algebra Appl. 267 (1997), 317-334.

[9] T. Kato, Perturbation Theory for Linear Operators, Grundlehren der mathematischen Wissenschaften 132 (Springer, Berlin, 1980).

[10] R. Kippenhahn, 'Über den Wertevorrat einer Matrix', Math. Nachr. 6 (1951), 193-228.

[11] P. Lancaster, 'On eigenvalues of matrices dependent on a parameter', Numer. Math. 6 (1964), 377-387.

[12] - Theory of Matrices (Academic Press, New York, 1969).

[13] F. D. Murnaghan, 'On the field of values of a square matrix', Proc. Nat. Acad. Sci. U.S.A. 18 (1932), 246-248.

[14] M. C. Pease III, Methods of Matrix Algebra (Academic Press, New York, 1965).

[15] F. Rellich, 'Störungstheorie der Spektralzerlegung I', Math. Ann. 113 (1937), 600-619.

[16] I. R. Shafarevich, Basic Algebraic Geometry (Springer, New York, 1977).

[17] M. Spivak, A Comprehensive Introduction to Differential Geometry, volume V, 2nd edition (Publish or Perish, Inc., Wilmington, 1979).

Fachbereich Mathematik

Technische Universität Berlin

Strasse des 17. Juni 136

D-10623 Berlin

Germany
School of Mathematics The University of New South Wales Sydney, NSW 2052 Australia 